\title{
Constraining spin-one color-octet resonances using CDF and ATLAS data
}

\author{
Alfonso R. Zerwekh ${ }^{\mathrm{a}}$ \\ Instituto de Física, Facultad de Ciencias, Universidad Austral de Chile and Centro Científico-Tecnológico de Valparaíso, Casilla 567, Valdivia, \\ Chile
}

Received: 26 August 2010 / Revised: 21 September 2010 / Published online: 24 November 2010

(C) The Author(s) 2010. This article is published with open access at Springerlink.com

\begin{abstract}
In this paper, we study the production of spinone color-octet resonances (colorons) at hadron colliders in a model independent way. We use dijets data measured by $\mathrm{CDF}$ (at $\sqrt{s}=1.96 \mathrm{TeV}$ and $\mathcal{L}=1.13 \mathrm{pb}^{-1}$ ) and ATLAS (at $\sqrt{s}=7 \mathrm{TeV}$ and $\mathcal{L}=315 \mathrm{nb}^{-1}$ ) collaborations at the Tevatron and the LHC respectively to impose limits on the coupling of colorons to fermions. We show that CDF data still produce the more stringent limits on the coloron coupling constant.
\end{abstract}

\section{Introduction}

Hadron colliders are the right tools for exploring new energy scales searching for new Physics. During the last decades this role has been played by the Tevatron. The discovery of the top quark $[1,2]$, with its originally unexpected huge mass is only one example of its significant contribution to our understanding of Particle Physics. Now the LHC is running and we hope that it will uncover phenomena beyond the Standard Model. The observation of New Physics is not, however, the only contribution of a collider. While new particles escape detection, the experimental data can be used to constrain models. Theorists have been extremely creative proposing extensions of the Standard Model and only direct or indirect experimental information can guide us to the path chosen by Nature.

Many proposed models predicts the existence of spinone color-octet particles. They have been studied, for example, in non-minimal technicolor (color-octet technirho, $\rho_{8}$ ) [3-7], topcolor (coloron) [8, 9], models with extra dimensions (Kaluza-Klein excitation of the gluon) [10] and chiral color models (axigluon) [11-14] but also in a model independent way [15-17]. It is generally expected that this kind of particles, which we will call generically "colorons", if

a e-mail: alfonsozerwekh@uach.cl they exist, must be copiously produced at hadron colliders due to their color charge and they must appear as resonances in the dijet spectrum. Now, we dispose of the first data of the ATLAS search of resonances that can decay into dijets [18] and we felt it is pertinent to upgrade our studies on spinone color-octet resonances and compare the limits obtained from ATLAS data with the ones obtained from CDF [19] measurements.

In Sect. 2 we recall a general description of colorons based exclusively on gauge symmetry and we show that a spin-one color-octet resonance cannot couple to two gluons by a dimension four operator and that the coupling constant of the coloron to quarks is not completely fixed by gauge symmetry.

In Sect. 3 we show the limits imposed by experimental data on the coloron coupling constant and we conclude that the more stringent limits are still obtained from CDF measurements.

Finally, in Sect. 4, we discuss our results.

\section{Theoretical description}

We start considering two vector fields $\left(A_{1 \mu}^{a}\right.$ and $\left.A_{2 \mu}^{a}\right)$ in the adjoint representation of the color group $S U(3)_{c}$ and we work in a basis where both fields transform like gauge fields. With these ingredients we can write down the following gauge invariant Lagrangian:

$$
\begin{aligned}
\mathcal{L}= & -\frac{1}{4} F_{1 \mu \nu}^{a} F_{1}^{a \mu \nu}-\frac{1}{4} F_{2 \mu \nu}^{a} F_{2}^{a \mu \nu} \\
& +\frac{M^{2}}{2 g_{2}^{2}}\left(g_{1} A_{1 \mu}^{a}-g_{2} A_{2 \mu}^{a}\right)^{2}
\end{aligned}
$$

where $M$ is a new mass scale present in the model. At this point it is necessary to remark that the mass term introduced in our Lagrangian is completely consistent with gauge invariance since, as it is straightforward to prove, the linear 
combination $\left(g_{1} A_{1 \mu}^{a}-g_{2} A_{2 \mu}^{a}\right)$ transforms homogeneously under local gauge transformations $[5,7,21]$.

The mass matrix originated by this Lagrangian (which is in this case the most general mass matrix for the gauge sector compatible with gauge symmetry) is exactly diagonalizable. In fact, the mass eigenstates fields can be written as

$$
\begin{aligned}
& G=A_{1} \cos (\alpha)+A_{2} \sin (\alpha) \\
& V=-A_{1} \sin (\alpha)+A_{2} \cos (\alpha)
\end{aligned}
$$

Here, for simplicity, we have dropped the Lorentz and color indexes and we have defined $\sin (\alpha)=g / g_{2}$ and $g$ is:

$g=\frac{g_{1} g_{2}}{\sqrt{g_{1}^{2}+g_{2}^{2}}}$

The masses of the physical states are

$m_{G}=0$

$m_{V}=\frac{M}{\cos (\alpha)}$

so we see that the Lagrangian describes, in fact, a massless gauge boson and a massive spin-one resonance in the adjoint representation. Clearly, we identify $G$ with the physical gluon and the massive state $V$ is the coloron

Let us study, for a moment, the decay process $V \rightarrow G G$. The relevant part of the Lagrangian, written in terms of the physical fields, is:

$$
\begin{aligned}
\mathcal{L}_{V G G}= & \left(-g_{1} \cos ^{2}(\alpha) \sin (\alpha)+g_{2} \cos (\alpha) \sin ^{2}(\alpha)\right) f^{a b c} \\
& \cdot\left\{\partial_{\mu} G_{\nu}^{a} G^{b \mu} V^{c \nu}++\partial_{\mu} G_{\nu}^{a} V^{b \mu} G^{c \nu}\right. \\
& \left.+\partial_{\mu} V_{\nu}^{a} G^{b \mu} G^{c v}\right\}
\end{aligned}
$$

It is clear that, due to the definition of $\alpha, g_{1} \cos (\alpha)=$ $g_{2} \sin (\alpha)$. Using this identity, we can see that the coupling constant of the $V G G$ interactions vanishes exactly $[5,9,16]$. This result is an important consequence of the gauge symmetry of the model. It is necessary to say, however, that this coupling can be restored by dimension 6 operators $[16,20]$. Nevertheless we will not consider here this case.

Let us turn our attention to the coupling with quarks. Because we have now two fields that transform as gauge fields $\left(A_{1 \mu}^{a}\right.$ and $A_{2 \mu}^{a}$ ) any combination of the form:

$g_{1}(1-k) A_{1 \mu}^{a}+g_{2} k A_{2 \mu}^{a}$

where $k$ is an arbitrary parameter, can be used to construct a covariant derivative $[7,21]$. Notice that the resulting generalized covariant derivative gives origin to a direct coupling, between the field $A_{2 \mu}$ and the fermions, parameterized by the new constant $k$. In other words, now $A_{2 \mu}$ couples to fermions not only through its mixing with $A_{1 \mu}$ (parameterized by the angle $\alpha$ ) but also through the new term introduced into the covariant derivative. Previously, we have used this kind of direct coupling in order to study the phenomenology of the color-octet technirho [7], it has been also useful in allowing us to propose a mechanism for reducing axigluon couplings [14] and recently this kind of terms played a crucial role in the construction of a model with two composite Higgs bosons [22]. Interestingly, this theoretical construction, i.e. the introduction of a linear combination of vector fields like (5), which depends on the arbitrary parameter $k$, naturally arises in deconstruction theories with "delocalized" fermions [23, 24].

As a consequence of the generalized covariant derivative, the coupling of the physical (mass eigenstates) vector bosons to quarks can be written as

$$
\begin{aligned}
\mathcal{L}_{V \bar{q} q}= & g_{\mathrm{QCD}} \frac{\lambda^{a}}{2} \bar{\psi} G_{\mu}^{a} \gamma^{\mu} \psi \\
& +g_{\mathrm{QCD}} \tan \alpha\left(\frac{k}{\sin ^{2} \alpha}-1\right) \frac{\lambda^{a}}{2} \bar{\psi} V_{\mu}^{a} \gamma^{\mu} \psi
\end{aligned}
$$

where $g_{\mathrm{QCD}}=g / \sqrt{2}$. Notice that the gluon couples to quarks in the usual way while the coloron coupling constant $\left(g_{V}=g_{\mathrm{QCD}} \tan \alpha\left(k / \sin ^{2} \alpha-1\right)\right)$ depends not only on the mixing angle but also on the arbitrary parameter $k$ and hence its value is not fixed by the gauge principle. This fact allows us to try to use experimental data in order to constrain the value of $g_{V}$ and make the coloron invisible to present searching efforts.

\section{Results}

We implemented the model described in the previous section into CalcHEP [25, 26] and use it to generate events and compute cross sections for the coloron production and decay into two jets, which could be compared to experimental data. Notice that, since the coloron does not couple to two gluons, as was shown above, we only have quarks in the initial and final states. Two set of events were generated: the first one using $\sqrt{s}=7.0 \mathrm{TeV}$ and the following set of cuts for comparing with ATLAS data:

$p_{T}^{j_{1}}>80.0 \mathrm{GeV}$

$p_{T}^{j_{2}}>30.0 \mathrm{GeV}$

$\left|\eta_{i}\right|<1.3$ or $1.8<\left|\eta_{i}\right|<2.5$

where $\eta_{i}$ is the pseudo-rapidity of jet $i$ and $j_{1}$ represents the jet with higher transversal momentum. For the second one, we use $\sqrt{s}=1.96 \mathrm{TeV}$ and $\left|\eta_{i}\right|<1.0$ in order to be compared with CDF data. For all the computations we 


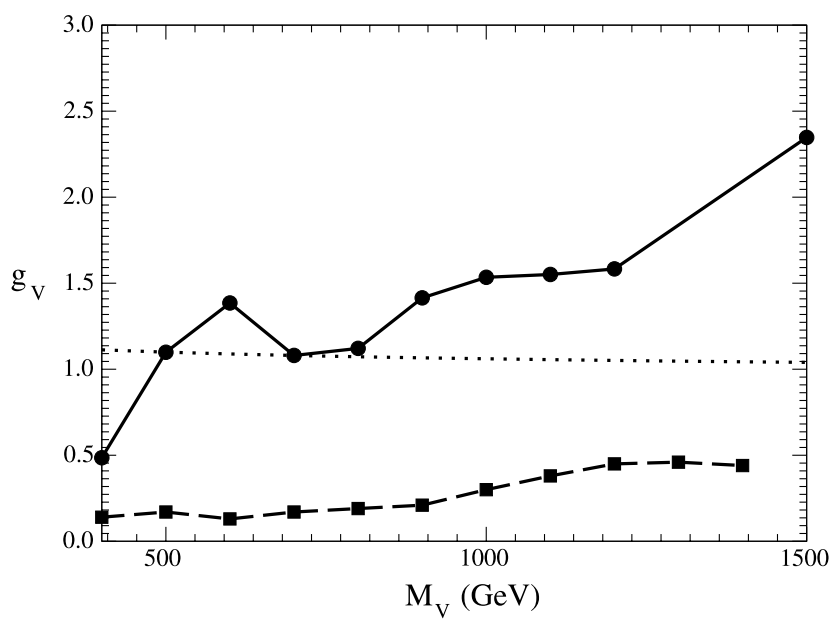

Fig. 1 Upper limits on the coloron coupling constant as a function of coloron mass obtained by demanding escape detection by ATLAS (dots with continuous line) and CDF (squares and dashed line). As a reference, we include in the figure the value of the (running) QCD coupling constant (dotted line)

use the CTEQ61 parton distribution function [27]. To obtain our limits we vary both the coloron mass $\left(M_{V}\right)$ and the coloron coupling constant $\left(g_{V}\right)$ in the intervals $M_{V} \in$ $[400,1500] \mathrm{GeV}$ and $g_{V} \in[0.05,2.50]$.

Our results are shown in Fig. 1. The dots with continuous line represent the maximum value of $g_{V}$ compatible with the 95\% C.L. upper limits obtained by ATLAS at $\sqrt{s}=7.0 \mathrm{TeV}$ and $\mathcal{L}=315 \mathrm{nb}^{-1}$. On the other hand, the squares with dashed line is similar but was obtained by comparing our predicted cross sections with the 95\% C.L. upper limits reported by CDF for $\sqrt{s}=1.96 \mathrm{TeV}$ and $\mathcal{L}=1.13 \mathrm{pb}^{-1}$. As a reference, we include in the figure the value of the (running) QCD coupling constant (dotted line).

As we can see, a spin-one color-octet resonance with QCD coupling (that is $g_{V}=g_{\mathrm{QCD}}$ ) is excluded by CDF but not by present ATLAS data (except by ATLAS in a small region near $M_{V}=400 \mathrm{GeV}$ ). In brief, our results show that CDF data still produce the more stringent limits on $g_{V}$.

It is worth to be remarked that despite the fact that our theoretical description, which justifies one to set limits on $g_{V}$ since its value is not fixed, was done for a color-octet field that has a vector coupling to quarks, a similar construction can be done in the case of chiral color models [14], and in principle our limits are also applicable to the axigluon.

\section{Conclusions}

In this work we have studied the production of color-octet spin-one resonances at the Tevatron and the LHC and its decay in dijets. We have compared our predicted cross section for several values of $g_{V}$ and $M_{V}$ with available upper limits from CDF and ATLAS measurements. In this form, we were able to set up upper limits on the value of $g_{V}$ in order to make those resonances invisible for the above cited experiments. We find that the most stringent limits still come from CDF data. Two reasons seems to determine this result. First, as we have shown, this kind of resonance is only produced by quark-anti-quark initial states. Being the Tevatron a $p \bar{p}$ collider the production of a coloron (or even an axigluon) would be favored in this collider. In other words, the Tevatron would be a more propitious environment for searching color-octet spin-one resonances. On the other hand, the ATLAS data used still reflect a low level of luminosity $\left(\mathcal{L}=315 \mathrm{nb}^{-1}\right)$. We hope that in the near future, when data with higher integrated luminosity become available, those limits can be improved.

Finally, we wish to emphasize that, as was pointed out elsewhere [7, 15], the LHC offers the unique opportunity of studying the production of a pair of colorons. This process is theoretically cleaner (almost model independent) and phenomenologically interesting.

Acknowledgement This work is partially supported by Fondecyt grant 1070880 and by the Conicyt grant "Southern Theoretical Physics Laboratory" ACT-91. TGD.

Open Access This article is distributed under the terms of the Creative Commons Attribution Noncommercial License which permits any noncommercial use, distribution, and reproduction in any medium, provided the original author(s) and source are credited.

\section{References}

1. F. Abe et al. (CDF Collaboration), Observation of top quark production in $\bar{p} p$ collisions. Phys. Rev. Lett. 74, 2626 (1995). arXiv:hep-ex/9503002

2. S. Abachi et al. (D0 Collaboration), Search for high mass top quark production in $p \bar{p}$ collisions at $\sqrt{s}=1.8 \mathrm{TeV}$. Phys. Rev. Lett. 74, 2422 (1995). arXiv:hep-ex/9411001

3. E. Eichten, I. Hinchliffe, K.D. Lane, C. Quigg, Super collider physics. Rev. Mod. Phys. 56, 579 (1984). Addendum-ibid. 58, 1065 (1986)

4. K.D. Lane, M.V. Ramana, Walking technicolor signatures at hadron colliders. Phys. Rev. D 44, 2678 (1991)

5. A.R. Zerwekh, R. Rosenfeld, Gauge invariance, color-octet vector resonances and double technieta production at the Tevatron. Phys. Lett. B 503, 325 (2001). arXiv:hep-ph/0103159

6. A.R. Zerwekh, Dijets production in nonminimal technicolor and limits on the color-octet technirho mass. Int. J. Mod. Phys. A 19, 4387 (2004)

7. A.R. Zerwekh, Consequences of partial vector meson dominance for the phenomenology of colored technihadrons. Eur. Phys. J. C 49, 1077 (2007). arXiv:hep-ph/0603094

8. R.S. Chivukula, A.G. Cohen, E.H. Simmons, New strong interactions at the Tevatron? Phys. Lett. B 380, 92 (1996). arXiv:hep-ph/9603311

9. E.H. Simmons, Coloron phenomenology. Phys. Rev. D 55, 1678 (1997). arXiv:hep-ph/9608269

10. B. Lillie, L. Randall, L.T. Wang, The bulk RS KK-gluon at the LHC. JHEP 0709, 074 (2007). arXiv:hep-ph/0701166

11. P.H. Frampton, S.L. Glashow, Unifiable chiral color with natural gim mechanism. Phys. Rev. Lett. 58, 2168 (1987) 
12. P.H. Frampton, S.L. Glashow, Chiral color: an alternative to the standard model. Phys. Lett. B 190, 157 (1987)

13. P. Ferrario, G. Rodrigo, Phys. Rev. D 80, 051701 (2009). arXiv:0906.5541 [hep-ph]

14. A.R. Zerwekh, Axigluon couplings in the presence of extra color-octet spin-one fields. Eur. Phys. J. C 65, 543 (2010). arXiv:0908.3116 [hep-ph]

15. B.A. Dobrescu, K. Kong, R. Mahbubani, Massive color-octet bosons and pairs of resonances at hadron colliders. Phys. Lett. B 670, 119 (2008). arXiv:0709.2378 [hep-ph]

16. C. Kilic, T. Okui, R. Sundrum, JHEP 0807, 038 (2008). arXiv: 0802.2568 [hep-ph]

17. C. Kilic, T. Okui, R. Sundrum, JHEP 1002, 018 (2010). arXiv: 0906.0577 [hep-ph]

18. T. A. Collaboration, Search for new particles in two-jet final states in $7 \mathrm{TeV}$ proton-proton collisions with the ATLAS detector at the LHC. arXiv:1008.2461 [hep-ex]

19. T. Aaltonen et al. (CDF Collaboration), Search for new particles decaying into dijets in proton-antiproton. Phys. Rev. D 79, 112002 (2009). arXiv:0812.4036 [hep-ex]
20. R.S. Chivukula, A. Grant, E.H. Simmons, Two gluon coupling and collider phenomenology of color octet technirho mesons. Phys. Lett. B 521, 239 (2001). arXiv:hep-ph/0109029

21. A.R. Zerwekh, Effective description of a gauge field and a tower of massive vector resonances. arXiv:hep-ph/0307130

22. A.R. Zerwekh, Mod. Phys. Lett. A 25, 423 (2010). arXiv: 0907.4690 [hep-ph]

23. R.S. Chivukula, E.H. Simmons, H.J. He, M. Kurachi, M. Tanabashi, Phys. Rev. D 71, 115001 (2005). arXiv:hep-ph/0502162

24. R.S. Chivukula, E.H. Simmons, H.J. He, M. Kurachi, M. Tanabashi, Phys. Rev. D 72, 015008 (2005). arXiv:hep-ph/0504114

25. A. Pukhov et al., CompHEP: a package for evaluation of Feynman diagrams and integration over multi-particle phase space. User's manual for version 33. arXiv:hep-ph/9908288

26. A. Pukhov, Calchep 2.3: MSSM, structure functions, event generation, 1, and generation of matrix elements for other packages. arXiv:hep-ph/0412191

27. J. Pumplin, D.R. Stump, J. Huston, H.L. Lai, P.M. Nadolsky, W.K. Tung, New generation of parton distributions with uncertainties from global QCD analysis. JHEP 0207, 012 (2002). arXiv:hep-ph/0201195 